\title{
Torus knots obtained by twisting torus knots
}

\author{
SANGYOP LEE
}

\begin{abstract}
A twisted torus knot is obtained from a torus knot by adding a number of full twists to some adjacent strands of the torus knot. In this paper, we show that if a twisted torus knot is a torus knot, then the number of added full twists is generically at most two in absolute value. We also show that this bound is the best possible by classifying twisted torus knots for which the upper bound is attained.
\end{abstract}

$57 \mathrm{~N} 10$

\section{Introduction}

Let $p, q$ be relatively prime positive integers and let $T(p, q)$ denote a torus knot in $S^{3}$ of type $(p, q)$; that is, $T(p, q)$ is a knot on the boundary of a standard solid torus in $S^{3}$, wrapping around the solid torus $p$ times in the longitudinal direction and $q$ times in the meridional direction. Let $V_{1}$ be the solid torus and $V_{2}$ the complementary solid torus; that is, $S^{3}=V_{1} \cup V_{2}$ and $V_{1} \cap V_{2}$ is a torus containing $T(p, q)$. Take an unknotted circle $C$ wrapping around $r(2 \leq r \leq p+q)$ adjacent strands of $T(p, q)$ and intersecting each solid torus $V_{i}(i=1,2)$ in a $\partial$-parallel arc. For any nonzero integer $s$, the result of $(-1 / s)$-surgery on $C$ is $S^{3}$ again. (For surgery coefficients, we refer to Rolfsen [16].) However, the surgery changes $T(p, q)$ into a new knot, called a twisted torus knot and written as $T(p, q, r, s)$. See the first picture in Figure 6, which illustrates $T(8,3,5,-2)$, and see also Lee [10] for a more detailed definition.

Twisted torus knots are interesting in both Dehn surgery and Heegaard splitting theory. These knots were introduced by Dean [3; 4] for his study on Seifert-fibered surgery on knots. Also, these knots were used to give examples of knots having the super additivity of tunnel number under connected sum (see Morimoto, Sakuma and Yokota [14]). There are many other interesting results on twisted torus knots; see the references in $[10]$.

Recently, there has been interest in studying the knot types of twisted torus knots. It is completely determined which twisted torus knots are unknotted [10]. There are infinitely many twisted torus knots which are satellite knots (see Morimoto and Yamada [15] and Lee [8]), but it is known that the number of added full twists must be small (see Lee [9]). 
In this paper, we are interested in determining four numbers $(p, q, r, s)$ for which $T(p, q, r, s)$ is a torus knot. Note that if $r=p$ then the knot type of $T(p, q)$ is easy to determine: in this case, if $(p, q, s)=(n, n-1,-1)$ for some integer $n$ then $T(p, q, r, s)$ is a trivial knot, and otherwise it a torus knot. Because the knot type of $T(p, q, r, s)$ is completely determined in $[8 ; 10]$ when $r$ is divisible by $q$, we focus on the case where $r \neq p$ and $r$ is not a multiple of $q$.

Theorem 1.1 Let $p, q, r, s$ be integers satisfying the following conditions:

- $\quad p, q$ are relatively prime, and $1 \leq q<p$.

- $2 \leq r \leq p+q, r$ is not a multiple of $q$, and $r \neq p$.

- $s \neq 0$.

Suppose that $T(p, q, r, s)$ is a torus knot. Then $|s| \leq 2$. Moreover, if $|s|=2$ then $(p, q, r, s)=(2 n \pm 1, n, n \pm 1,-2)$ and $T(p, q, r, s)=T(2 n \pm 1, \mp 2)$ for some positive integer $n$.

Combining Theorem 1.1 with the main results in [9] and [10] we obtain the following corollary.

Corollary 1.2 Let $p, q, r, s$ be as in Theorem 1.1 and suppose that $|s| \geq 3$. Then $T(p, q, r, s)$ is a hyperbolic knot.

Some figures in this paper are best viewed in color: readers are recommended to view them in an electronic version.

\section{Lemmas}

Throughout this paper, we write $K=T(p, q)$ and $K^{\prime}=T(p, q, r, s)$, and we assume that $K^{\prime}$ is a torus knot. Note that $q \geq 2$ by the hypothesis that $r$ is not a multiple of $q$. Let $V_{1}, V_{2}, C$ be as in the previous section. Let $M=S^{3}-\operatorname{int} N(K \cup C)$ and $W_{i}=V_{i} \cap M=V_{i}-\operatorname{int} N(K \cup C)$ for $i=1,2$. Then $M$ is a compact 3-manifold bounded by two boundary tori, say $\partial_{K} M(=\partial N(K))$ and $\partial_{C} M(=\partial N(C))$, and $W_{i}$ is a genus two handlebody for each $i=1,2$. Let $\partial_{X} W_{i}=\partial_{X} M \cap W_{i}$ for each $X=K, C$. Then $\partial_{X} W_{i}$ is an annulus in $\partial W_{i}$ and its core $X_{i}$ is a simple closed curve in $\partial W_{i}$.

Given a 3 -manifold $Q$ with a simple closed curve $\ell$ in $\partial Q$, we use $Q[\ell]$ to denote the result of adding a $2-$ handle to $Q$ along the curve $\ell$. Note that $W_{i}\left[C_{i}\right]$ is the solid torus $V_{i}$. 
For slopes $\alpha_{X}$ in $\partial_{X} M$, we define $M\left(K, C ; \alpha_{K}, \alpha_{C}\right)$ to be the 3 -manifold obtained from $M$ by gluing a solid torus along $\partial_{X} M$ in such a way that $\alpha_{X}$ bounds a meridian disk of the solid torus for each $X=K, C$. We similarly define $M\left(\alpha_{C}\right)$ to be the result of gluing a solid torus only on $\partial_{C} M$ with slope $\alpha_{C}$.

Lemma 2.1 For each $i=1,2$, either $W_{i}\left[K_{i}\right]$ is $\partial$-irreducible or it is a solid torus. Moreover, the following hold.

(1) $W_{1}\left[K_{1}\right]$ is a solid torus if and only if $r \equiv \pm 1$ or $\pm q \bmod p$.

(2) $W_{2}\left[K_{2}\right]$ is a solid torus if and only if $r \equiv \pm 1$ or $\pm p \bmod q$.

Proof We break $\left(S^{3}, C\right)$ up into $\left(V_{1}, V_{1} \cap C\right)$ and $\left(V_{2}, V_{2} \cap C\right)$. Consider the case $i=2$ only. (The case $i=1$ is similar.) We first show that we may assume $r<p$. Suppose that $r>p$. Then $V_{2} \cap C$ is a $\partial$-parallel arc in $V_{2}$ running over $r(>p)$ strands of $K$. By applying an isotopy in $V_{2}$ as shown in Figure 1, which illustrates the case $(p, q, r)=(7,5,8)$, one can deform $V_{2} \cap C$ to an arc running over $r^{\prime}(<p)$ strands of $K$. Note that $r^{\prime} \equiv r \bmod q$.
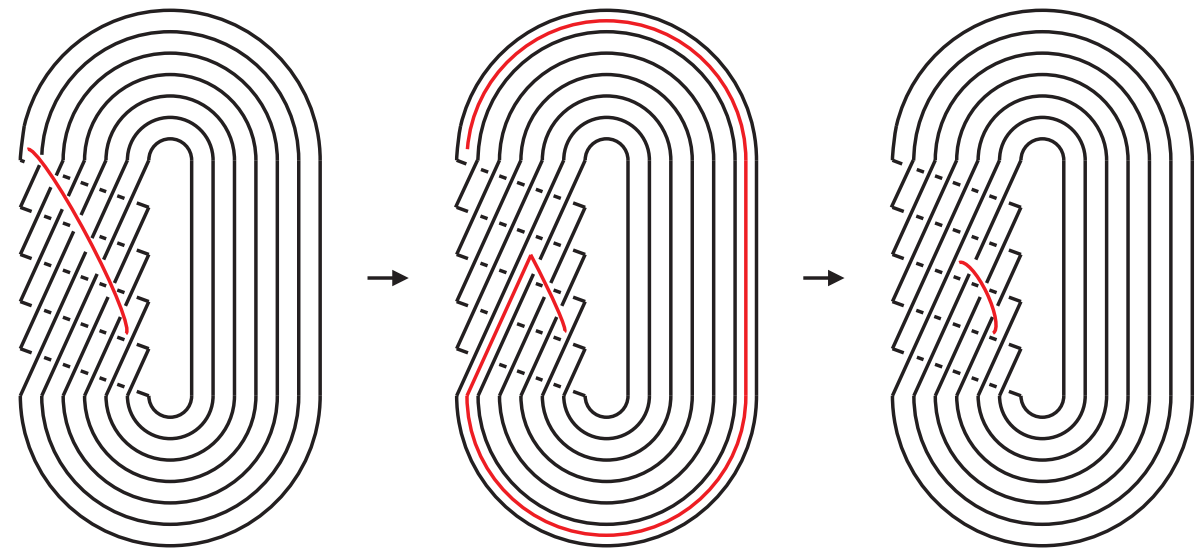

Figure 1

For the remainder of this proof, we assume $r<p$. Consider the knots $K^{\prime \prime}, C^{\prime \prime}$ in Figure 2 lying on a genus two Heegaard surface $\Sigma$ which splits $S^{3}$ into two handlebodies $H_{1}, H_{2}$. Using [10, Lemma 6.4], one can see that $H_{2}\left[K^{\prime \prime}\right]$ is homeomorphic to $W_{2}\left[K_{2}\right]$. (There is a typo in the statement of the lemma: " $H_{i}-\operatorname{int}\left(K^{\prime} \cup C^{\prime}\right)$ " in the third line must be replaced by " $H_{i}-\operatorname{int}\left(N\left(K^{\prime}\right) \cup N\left(C^{\prime}\right)\right)$ ".) It follows from [4, Theorem 3.4] that $W_{2}\left[K_{2}\right]$ is a solid torus if and only if $r \equiv \pm 1$ or $\pm p \bmod q$.

In order to complete the proof, we need only to show that if $W_{2}\left[K_{2}\right]$ is $\partial$-reducible then it is a solid torus. Suppose that $W_{2}\left[K_{2}\right] \cong H_{2}\left[K^{\prime \prime}\right]$ is $\partial$-reducible. By Jaco's handle 


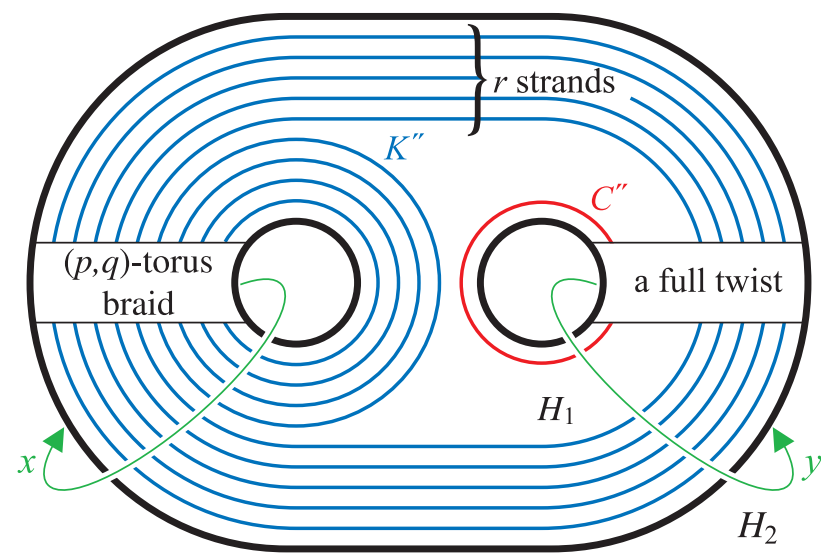

Figure 2

addition theorem [7], $\partial \mathrm{H}_{2}-\mathrm{K}^{\prime \prime}$ is compressible in $\mathrm{H}_{2}$. We can take a nonseparating compressing disk for $\partial \mathrm{H}_{2}-K^{\prime \prime}$. Cutting $\mathrm{H}_{2}$ along the compressing disk, we get a solid torus whose boundary contains $K^{\prime \prime}$.

If $K^{\prime \prime}$ is isotopic to a core of the solid torus, then $W_{2}\left[K_{2}\right] \cong H_{2}\left[K^{\prime \prime}\right]$ is a solid torus and we are done. Suppose otherwise. Then $K^{\prime \prime}$ represents a proper power of a primitive element of $\pi_{1}\left(H_{2}\right)$ (note that $\pi_{1}\left(H_{2}\right)$ is a free group on two generators, say $x, y$ in Figure 2). After the possible replacement of $x$ by $x^{-1}$ or $y$ by $y^{-1}$, we may assume that the exponents of the primitive element are all positive. It is known that a primitive element of a rank two free group with positive exponents has one generator which appears solely with exponent +1 , and that the primitive element can also be cyclically permuted so that the exponents of the other generator differ by at most one [2]; it is easy to see that a proper power of a primitive element also has this property. The proof of [4, Theorem 3.4] was completed by using only this property of primitive elements and hence the proof goes through verbatim to show that $K^{\prime \prime}$ represents a proper power of a primitive element of the free group $\pi_{1}\left(H_{2}\right)$ if and only if $r \equiv \pm 1$ or $\pm p \bmod q$. However, the necessary condition holds only if $K^{\prime \prime}$ represents a primitive element of $\pi_{1}\left(H_{2}\right)$ by [4, Theorem 3.4]. This implies that $K^{\prime \prime}$ cannot represent a proper power of a primitive element.

Lemma 2.2 If $|s| \geq 2$, then $K^{\prime}\left(p q+r^{2} s\right)$ is a Seifert fiber space with orbifold $S^{2}(p, q,|s|)$ and $W_{i}\left[K_{i}\right]$ is a solid torus for each $i=1,2$, where $K^{\prime}\left(p q+r^{2} s\right)$ is the 3-manifold obtained by Dehn surgery on $K^{\prime}$ with slope $p q+r^{2} s$.

Proof Let $J_{-1 / s}$ denote the solid torus glued in $M(K, C ; p q,-1 / s)$ along the torus $\partial_{C} M$. Note that slope $p q$ is the cabling slope associated to the torus knot $K$. Using a 
Rolfsen twist [16], one easily sees that

$$
K^{\prime}\left(p q+r^{2} s\right)=M(K, C ; p q,-1 / s)=W_{i}\left[K_{i}\right] \cup\left(W_{j}\left[K_{j}\right] \cup J_{-1 / s}\right),
$$

where $\{i, j\}=\{1,2\}$ and the union $W_{j}\left[K_{j}\right] \cup J_{-1 / s}$ is taken along the annulus $\partial_{C} W_{j}$ wrapping around the solid torus $J_{-1 / s}|s|$ times in its longitudinal direction. Let $T$ be the common boundary torus of $W_{i}\left[K_{i}\right]$ and $W_{j}\left[K_{j}\right] \cup J_{-1 / s}$.

We claim that $T$ is incompressible in $W_{j}\left[K_{j}\right] \cup J_{-1 / s}$. First, note that $W_{j}\left[K_{j}\right]\left[C_{j}\right]=$ $W_{j}\left[C_{j}\right]\left[K_{j}\right]=V_{j}\left[K_{j}\right]$ is a once-punctured lens space $L(p, q)$ or $L(q, p)$ depending on whether $j=1$ or $j=2$. Thus if $W_{j}\left[K_{j}\right]$ is a solid torus then $W_{j}\left[K_{j}\right] \cup J_{-1 / s}$ is a Seifert fiber space over the disk with two exceptional fibers because the annulus $\partial_{C} W_{j}$ wraps around each of the solid tori $W_{j}\left[K_{j}\right]$ and $J_{-1 / s}$ at least two times in the longitudinal direction. It follows that $T$ is incompressible in $W_{j}\left[K_{j}\right] \cup J_{-1 / s}$. Suppose that $W_{j}\left[K_{j}\right]$ is not a solid torus. Then $W_{j}\left[K_{j}\right]$ is $\partial$-irreducible by Lemma 2.1 and hence the annulus $\partial_{C} W_{j}$ is incompressible in $W_{j}\left[K_{j}\right]$. Also, the annulus is incompressible in $J_{-1 / s}$ since it wraps around $J_{-1 / s}$ at least two times in the longitudinal direction. Thus $T$ is incompressible in $W_{j}\left[K_{j}\right] \cup J_{-1 / s}$, since otherwise by the incompressibility of $\partial_{C} W_{j}$ there would be a compressing disk for $T$ lying entirely in either $W_{j}\left[K_{j}\right]$ or $J_{-1 / s}$ but missing $\partial_{C} W_{j}$.

No Dehn surgery on a torus knot yields a separating incompressible torus, so $T$ is compressible in $W_{i}\left[K_{i}\right]$ and by Lemma $2.1 W_{i}\left[K_{i}\right]$ must be a solid torus. Since the index $i$ can take any value 1 or 2 , both of $W_{1}\left[K_{1}\right]$ and $W_{2}\left[K_{2}\right]$ are solid tori. Since $W_{1}\left[K_{1}\right]\left[C_{1}\right]$ is a once-punctured lens space $L(p, q), W_{1}\left[K_{1}\right]$ is a fibered solid torus over $D^{2}(p)$ with $C_{1}$ a fiber. Similarly, $W_{2}\left[K_{2}\right]$ is a fibered solid torus over $D^{2}(q)$ with $C_{2}$ a fiber and hence $W_{2}\left[K_{2}\right] \cup J_{-1 / s}$ is a Seifert fiber space over $D^{2}(q,|s|)$ with $C_{2}$ a fiber. The two curves $C_{1}$ and $C_{2}$ are isotopic along the torus $\partial J_{-1 / s}$ in $W_{1}\left[K_{1}\right] \cup\left(W_{2}\left[K_{2}\right] \cup J_{-1 / s}\right)=K^{\prime}\left(p q+r^{2} s\right)$, hence the result follows.

\section{Lemma 2.3}

$$
|s| \leq 3
$$

Proof Recall that $r \neq p$ and $r$ is not a multiple of $q$. Thus $M$ is a hyperbolic 3manifold by [10, Proposition 5.7]. The manifolds $M(1 / 0)$ and $M(-1 / s)$, the exteriors of the torus knots $K$ and $K^{\prime}$, are annular, so we have $|s|=\Delta(1 / 0,-1 / s) \leq 5$ by [5]. Moreover, if $|s|=4$ or 5 , then both $M(1 / 0)$ and $M(-1 / s)$ must be toroidal (see the remark following [6, Theorem 1.1]). However, the exterior of a torus knot cannot be toroidal and hence $|s| \neq 4,5$ and the result follows.

A braid knot (in this paper a knot which is a closed braid will be called so) is a fibered knot if all of its crossings have the same sign [17] and its genus is easily calculated: 
if $b$ is the number of strands of the braid and $c$ is the number of crossings, then the Euler characteristic $\chi$ of a fiber surface and the genus $g$ of the knot are respectively given by $\chi=b-c$ and $g=(1-b+c) / 2$.

Lemma 2.4 If $|s| \geq 2$, then $s=-2$ or $s=-3$.

Proof Suppose that $s=2$ or 3 . We first claim that $T(p, q, r, s)$ is a positive braid knot, a braid knot with all crossings positive. If $r<p$, then $T(p, q, r, s)$ is the knot obtained by closing the braid shown in Figure 3(a), where the box with an integer pair $(a, b)$ denotes an $(a, b)$-torus braid. If $r>p$, then by performing an isotopy one can see that $T(p, q, r, s)$ is the knot obtained by closing the braid shown in Figure 3(b). For example, see Figure 4 which illustrates a sequence of isotopies for the case that $(p, q, r, s)=(7,5,9, s)$.

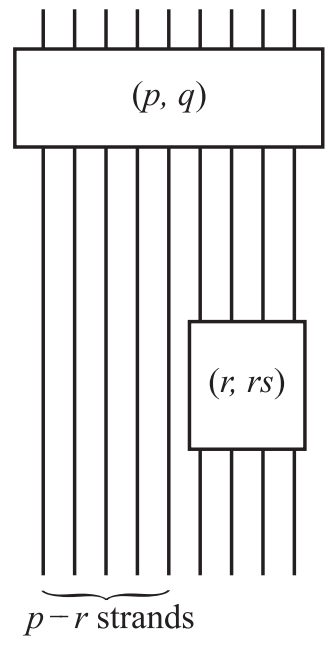

(a)

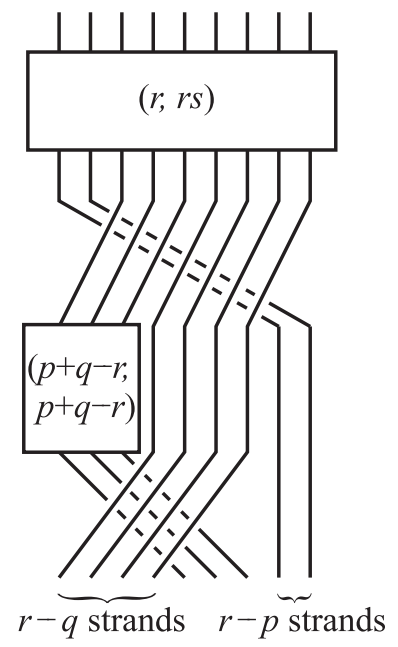

(b)

Figure 3

Since $T(p, q, r, s)$ is a positive braid knot, it is a fibered knot. Let $F$ be a fiber surface. Then $\chi(F)=b-c$, where $b$ is the number of strands of the closed positive braid presentation of $T(p, q, r, s)$ given in Figure 3 and $c$ is the number of crossings in the presentation. If $r<p$, then using Figure 3(a), one easily sees that $\chi(F)=$ $p-((p-1) q+(r-1) r s)$. If $r>p$, then from Figure 3(b) one gets $b=r$ and

$$
\begin{aligned}
c= & (r-1) r s+(r-p) p+(p+q-r-1)(p+q-r) \\
& +(p+q-r)(r-q)=r^{2} s-r s+p q-p-q+r,
\end{aligned}
$$



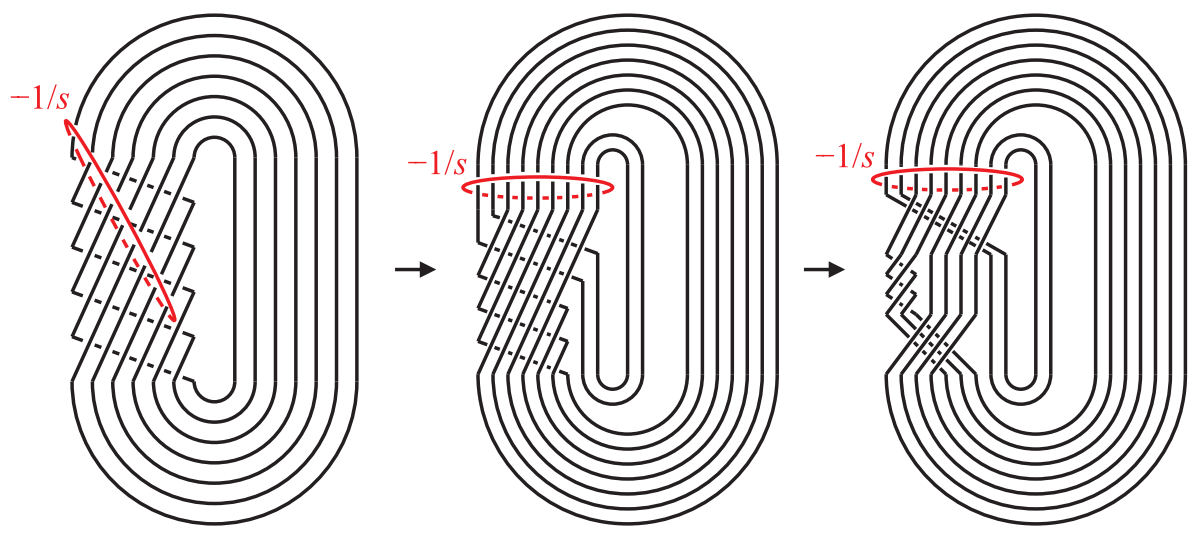

Figure 4

hence

$$
\chi(F)=r-\left(r^{2} s-r s+p q-p-q+r\right)=p-((p-1) q+(r-1) r s) .
$$

By Lemma 2.2, $K^{\prime}\left(p q+r^{2} s\right)$ is a Seifert fiber space with orbifold $S^{2}(p, q, s)$. A simple calculation shows that

$$
\left|p q+r^{2} s\right|-p-q-s+\chi(F)=(r-1) s>0 .
$$

This shows that $K^{\prime}=T(p, q, r, s)$ is not a torus knot by [4, Lemma 5.1], a contradiction.

Hereafter, we assume $s=-2$ or -3 . Since $K^{\prime}\left(p q+r^{2} s\right)$ is a Seifert fiber space with orbifold $S^{2}(p, q,|s|)$, there are six possibilities for the knot type of $K^{\prime}$,

$$
K^{\prime}=T(p, \varepsilon q), \quad T(p, \varepsilon s) \text { or } T(q, \varepsilon s),
$$

where $\varepsilon= \pm 1$. Also, if we let $K^{\prime}=T(x, \varepsilon y)$ and $\{x, y, z\}=\{p, q, s\}$, then $x, y$ and $z$ must satisfy

$$
\left|p q+r^{2} s-\varepsilon x y\right|=|z|
$$

\section{Lemma 2.5}

$$
r<p
$$

Proof Assume $r>p$. First, suppose $K^{\prime}=T(p, \varepsilon q)$. Then

$$
\left|p q+r^{2} s-\varepsilon p q\right|=|s| \text {. }
$$

This is impossible because

$$
\left|p q+r^{2} s-\varepsilon p q\right| \geq r^{2}|s|-2 p q>2 p^{2}-2 p q=2 p(p-q) \geq 4>|s| .
$$


Next, suppose $K^{\prime}=T(p, \varepsilon s)$. Then

$$
\left|p q+r^{2} s-\varepsilon p s\right|=q .
$$

This is also impossible because

$$
\begin{aligned}
\left|p q+r^{2} s-\varepsilon p s\right| & \geq r^{2}|s|-p q-p|s|>p(p|s|-q-|s|) \\
& >p(2 p-q-3)=p((p-q-1)+(p-2)) \geq p(p-2) \geq p>q .
\end{aligned}
$$

Finally, suppose $K^{\prime}=T(q, \varepsilon s)$. Then

$$
\left|p q+r^{2} s-\varepsilon q s\right|=p .
$$

This is also impossible because

$$
\begin{aligned}
\left|p q+r^{2} s-\varepsilon q s\right| \geq r^{2}|s|-p q-q|s| & >r^{2}|s|-p q-p|s| \\
& >p(p|s|-q-|s|)>p(2 p-q-3) \geq p .
\end{aligned}
$$

Therefore we must have $r<p$.

Lemma 2.6 If $p>2 q, r=p-q$ and $s<0$, then $T(p, q, r, s)$ is a fibered knot and its fiber surface has Euler characteristic $\chi$ given by the formula

$$
\chi=(p-q)-((p-q-1)(-s p+(s-2) q)+(p-2 q) q) .
$$

Proof Since $p>2 q, r=p-q>q$. There is an isotopy deforming $T(p, q, r, s)$ to the closure of the braid in Figure 5. See Figure 6, which illustrates the case that $p=8, q=3, r=5, s=-2$.

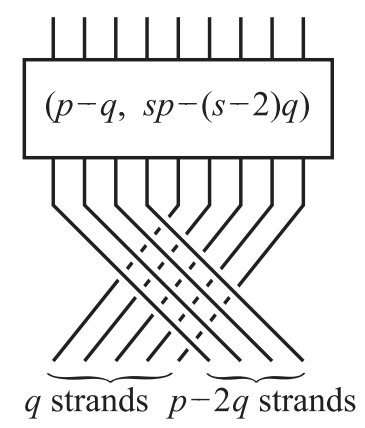

Figure 5

All crossings of the braid knot are negative, so the braid knot is fibered. Since the braid knot has $p-q$ strands and $(p-q-1)(-s p+(s-2) q)+(p-2 q) q$ crossings, the Euler characteristic $\chi$ of a fiber surface is given by the formula

$$
\chi=(p-q)-((p-q-1)(-s p+(s-2) q)+(p-2 q) q) .
$$




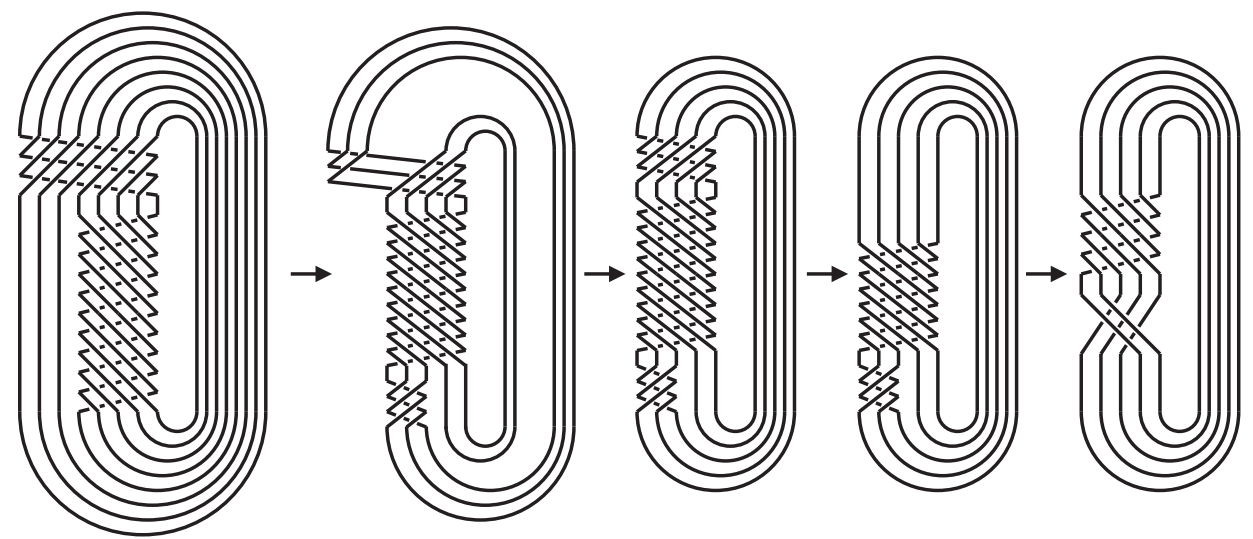

Figure 6

\section{The case $s=-3$}

We will show that $s \neq-3$ by ruling out all possibilities for $K^{\prime}$ listed in Equation (2-1). For the sake of contradiction we assume that $s=-3$ throughout this section.

Recall that given a knot, if an arc is added to the knot to form a spatial graph whose complement is an open handlebody of genus two, then the arc is called an unknotting tunnel for the knot. A knot in $S^{3}$ is called a $(1,1)-k n o t$ if there exists a decomposition of $S^{3}$ into two solid tori such that the intersection of the knot with each solid torus is a single $\partial$-parallel arc. A part of a core of each solid torus in the decomposition becomes an unknotting tunnel for the $(1,1)-$ knot when the arc of intersection of the knot with the solid torus is isotoped so as to meet the core in a subarc. Such an unknotting tunnel is called a $(1,1)$-tunnel.

Unknotting tunnels for torus knots were classified in [1] and [11]. Given a torus knot $T(a, b)$, there is a torus in $S^{3}$ containing $T(a, b)$ and splitting $S^{3}$ into two solid tori, inner and outer. The middle tunnel for $T(a, b)$ is represented by a spanning arc of a cabling annulus in the complement of $T(a, b)$. The inner/outer tunnel for $T(a, b)$ is represented by a properly embedded $\operatorname{arc} \alpha$ in the inner/outer solid torus such that the union of the arc with one of two parts of $T(a, b)$ cut off by $\partial \alpha$ is isotopic to a core of the solid torus. It is easy to see that the inner/outer tunnel for a torus knot is a $(1,1)$-tunnel.

To proceed, we need to prepare the following technical lemma.

Lemma 3.1 For (2)-(4) of the following statements, suppose that $n$ is any positive integer which is not a multiple of 3 . 
(1) $T(5,2,3,-3) \neq T(5,-3)$.

(2) $T(12,7,5,-3) \neq T(n, 3)$.

(3) $T(14,9,5,-3) \neq T(n, 3)$.

(4) $T(18,11,7,-3) \neq T(n, 3)$.

Proof (1) By an isotopy, one can deform $T(5,2,3,-3)$ to a 3-braid knot with 12 crossings, all having negative signs. See Figure 7 . Thus $T(5,2,3,-3)$ is a fibered knot and its genus is $\frac{1}{2}(1-3+12)=5$. However, the genus of $T(5,-3)$ is $\frac{1}{2}(5-1)(3-1)=4$, so $T(5,2,3,-3) \neq T(5,-3)$.

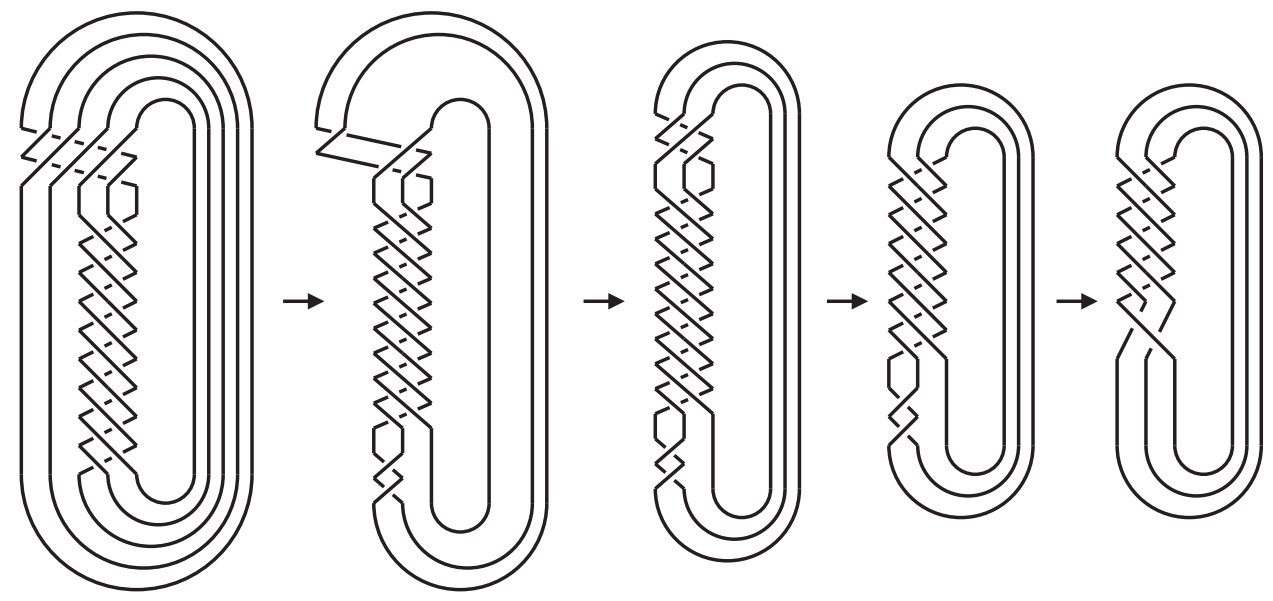

Figure 7

(2) Applying the same argument as in the proof of [3, Lemma 3.1.1], one can show that $T(a, b, c, d)$ and $T(b, a, c, d)$ are isotopic if $c<a$ and $c<b$. Hence $T(12,7,5,-3)=T(7,12,5,-3)$. Using a deformation, one can also see that the arc $\tau$ shown in Figure 8 (a) is an unknotting tunnel for $T(7,12,5,-3)$. Let $\gamma$ and $\gamma^{\prime}$ be two subarcs of $T(7,12,5,-3)$ cut off by $\partial \tau$. Consider two knots $\gamma \cup \tau$ and $\gamma^{\prime} \cup \tau$, which are shown in Figure 8 (b), (c). They are the twisted torus knots $T(4,7,3,-3)=T(7,4,3,-3)$ and $T(3,5,2,-3)=T(5,3,2,-3)$, which are nontrivial knots by [10, Theorem 1.1]. Since both knots $\gamma \cup \tau$ and $\gamma^{\prime} \cup \tau$ are nontrivial, $\tau$ is not a $(1,1)$-tunnel for $T(7,12,5,-3)$ by [13, Proposition 1.3]. However, it is easy to see that $T(n, 3)$ satisfies condition (iii) of [12, Theorem 3.3], so the middle tunnel for $T(n, 3)$ is isotopic to either inner or outer tunnel. It follows that any tunnel for $T(n, 3)$ is a $(1,1)$-tunnel. This implies that $T(12,7,5,-3)=T(7,12,5,-3) \neq T(n, 3)$.

(3), (4) For both $(a, b, c)=(14,9,5)$ and $(18,11,7)$, the torus knots $T(a, b, c,-3)$ and $T(b, a, c,-3)$ are isotopic. One can see that the arc $\tau$ in Figure 9 is an unknotting tunnel for $T(b, a, c,-3)$. For $(a, b, c)=(14,9,5)$, the tunnel $\tau$ splits 


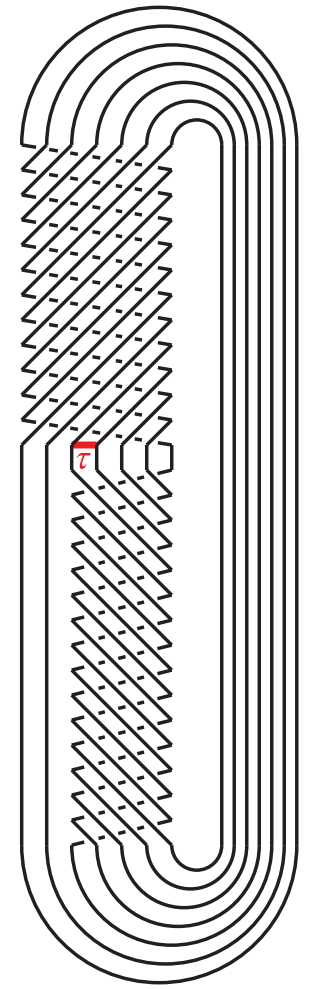

(a)

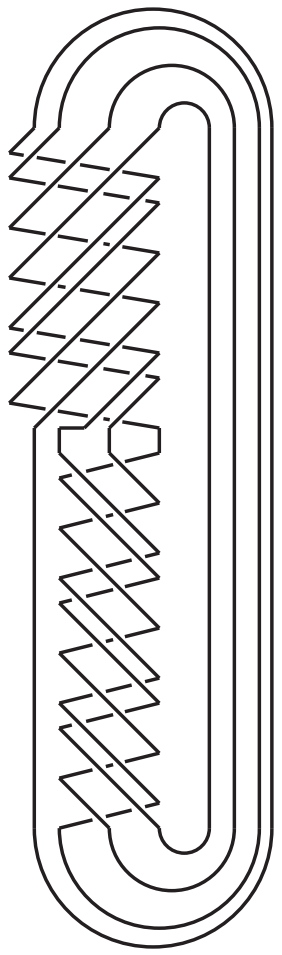

(b)

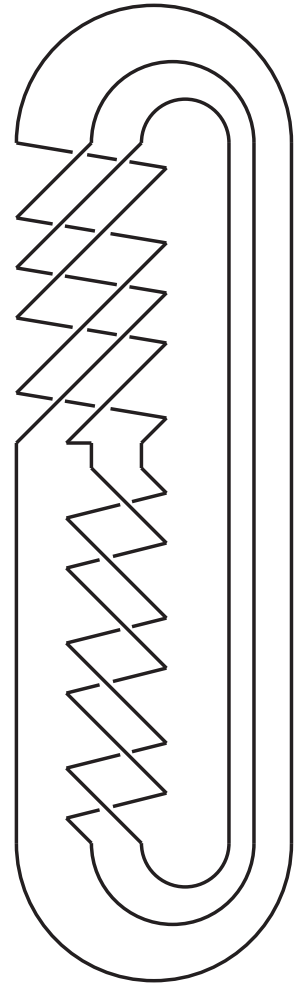

(c)

Figure 8

$T(b, a, c,-3)=T(9,14,5,-3)$ into two twisted torus knots $T(7,11,4,-3)$ and $T(2,3,1,-3)$, both of which are nontrivial knots by [10, Theorem 1.1] (note that $T(2,3,1,-3)$ is a trefoil knot). For $(a, b, c)=(18,11,7)$, then the tunnel splits $T(b, a, c,-3)=T(11,18,7,-3)$ into two twisted torus knots $T(8,13,5,-3)$ and $T(3,5,2,-3)$, both of which are nontrivial knots by [10, Theorem 1.1] again. Applying the same argument as in (2), one can show that $T(a, b, c,-3) \neq T(n, 3)$ for both $(a, b, c)=(14,9,5)$ and $(18,11,7)$.

By Lemma 2.2, $W_{1}\left[K_{1}\right]$ is a solid torus. By Lemmas 2.1 and 2.5, we have $r=p-1$ or $p-q$.

\section{Lemma 3.2}

$$
K^{\prime} \neq T(p, \varepsilon q) \text {. }
$$

Proof Assume for contradiction that $K^{\prime}=T(p, \varepsilon q)$. Then (2-2) implies

$$
\left|p q-3 r^{2}-\varepsilon p q\right|=3 \text {. }
$$




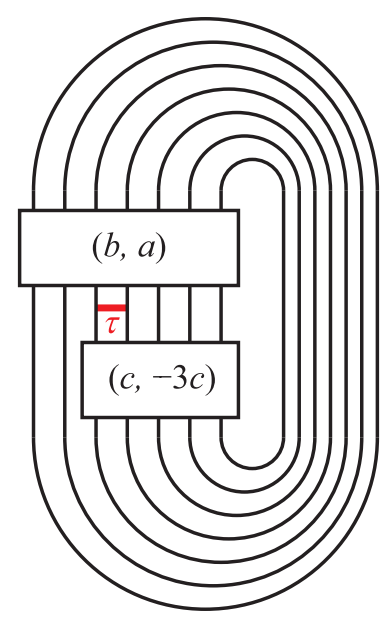

Figure 9

Since $r>1$ by hypothesis, we must have $\varepsilon=-1$. If $r=p-1$, then $p-q \geq 2$ (and hence $p \geq 4$ ) by the hypothesis that $r$ is not a multiple of $q$. Thus we get

$$
\begin{aligned}
\left|p q-3 r^{2}-\varepsilon p q\right|=3 p^{2}-6 p+3-2 p q & =p^{2}-6 p+3+2 p(p-q) \\
& \geq p^{2}-6 p+3+4 p=p(p-2)+3>3,
\end{aligned}
$$

which gives a contradiction.

Suppose $r=p-q$. From Equation (3-1) we get

$$
3(p-q)^{2}=2 p q \pm 3 .
$$

One can see that even the congruence equation $3(p-q)^{2} \equiv 2 p q-3 \bmod 4$ has no solution in integers $p, q$. Thus we must have

$$
3(p-q)^{2}=2 p q+3,
$$

which is equivalent to $3 p^{2}+3 q^{2}=8 p q+3$. Dividing both sides by $3 p q$, we get $p / q+q / p=8 / 3+1 /(p q)>8 / 3$. Noting that the function $t+1 / t$ is increasing for $t \geq 1$, one sees that $p / q>2$. By Lemma 2.6 our knot $T(p, q, r, s)$ is a fibered knot and

$$
\begin{aligned}
\chi(F) & =(p-q)-((p-q-1)(3 p-5 q)+(p-2 q) q) \\
& =(p-q)-\left(3 p^{2}-8 p q+3 q^{2}+p q-3 p+5 q\right) \\
& =(p-q)-(3+p q-3 p+5 q)=4 p-6 q-p q-3
\end{aligned}
$$


where $F$ is a fiber surface. Since our knot $K^{\prime}=T(p, q, r, s)$ was assumed to be the torus knot $T(p, \varepsilon q)$, we must have $\chi(F)=p+q-p q$ and hence $4 p-6 q-p q-3=$ $p+q-p q$, giving $3 p=7 q+3$. Solving the simultaneous equations

$$
\begin{aligned}
3(p-q)^{2} & =2 p q+3, \\
3 p & =7 q+3,
\end{aligned}
$$

we get $(p, q)=(1,0)$ or $(-20,-9)$, both of which are absurd.
Lemma 3.3
$K^{\prime} \neq T(p, 3 \varepsilon)$.

Proof Assume for the sake of contradiction that $K^{\prime}=T(p, 3 \varepsilon)$. Then

$$
\left|p q-3 r^{2}-3 \varepsilon p\right|=q .
$$

If $r=p-1$, then $r=p-1>p-2 \geq q$ and Equation (3-2) gives $(p \pm 1) q=$ $3 p^{2}-6 p+3+3 \varepsilon p$, so

$$
(p+1)(p-2) \geq(p \pm 1) q=3 p^{2}-6 p+3+3 \varepsilon p \geq 3 p^{2}-9 p+3,
$$

which yields $2(p-2)^{2} \leq 3$ and hence $p=3$, contradicting $p \geq q+2 \geq 4$.

Suppose $r=p-q$. From Equation (3-2) we get $3(p-q)^{2}-p q+3 \varepsilon p=\varepsilon^{\prime} q$, or equivalently

$$
p\left(q-3 \varepsilon+\varepsilon^{\prime}\right)=(p-q)\left(3 p-3 q+\varepsilon^{\prime}\right),
$$

where $\varepsilon^{\prime}= \pm 1$. Since $p$ and $p-q$ are relatively prime, $p$ divides $3 p-3 q+\varepsilon^{\prime}$ and hence it divides $3 q-\varepsilon^{\prime}$. Put $3 q-\varepsilon^{\prime}=k p(k=1,2)$. Then Equation (3-3) gives $p\left(q-3 \varepsilon+\varepsilon^{\prime}\right)=(p-q)(3-k) p$ or equivalently $q-3 \varepsilon+\varepsilon^{\prime}=\left(\left(3 q-\varepsilon^{\prime}\right) / k-q\right)(3-k)$. Solving this equation, we get

$$
q=\frac{-3 k \varepsilon+3 \varepsilon^{\prime}}{(3-k)^{2}-k} \quad \text { and } \quad p=\frac{3 q-\varepsilon^{\prime}}{k},
$$

which yields

$$
(p, q)= \begin{cases}(5,2) & \text { if }\left(k, \varepsilon, \varepsilon^{\prime}\right)=(1,-1,1) \\ (4,3) & \text { if }\left(k, \varepsilon, \varepsilon^{\prime}\right)=(2,1,1) \\ (14,9) & \text { if }\left(k, \varepsilon, \varepsilon^{\prime}\right)=(2,1,-1)\end{cases}
$$

Thus $K^{\prime}=T(5,2,3,-3)$ or $T(14,9,5,-3)$ because $r \neq 1$. This contradicts statements (1) and (3) of Lemma 3.1.

\section{Lemma 3.4}

$$
K^{\prime} \neq T(q, 3 \varepsilon)
$$


Proof Assume for the sake of contradiction that $K^{\prime}=T(q, 3 \varepsilon)$. Then

$$
\left|p q-3 r^{2}-3 \varepsilon q\right|=p .
$$

If $r=p-1$, then $r=p-1>p-2 \geq q$ and Equation (3-4) gives $(p-3 \varepsilon) q=$ $3 p^{2}-6 p \pm p+3$, so

$$
(p+3)(p-2) \geq(p-3 \varepsilon) q=3 p^{2}-6 p \pm p+3 \geq 3 p^{2}-7 p+3,
$$

which yields $2(p-2)^{2}+1 \leq 0$, a contradiction.

Suppose $r=p-q$. Then interchanging the roles of $p$ and $q$ in the proof of Lemma 3.3, one can see that

$$
p=\frac{-3 k \varepsilon+3 \varepsilon^{\prime}}{(3-k)^{2}-k} \quad \text { and } \quad q=\frac{3 p-\varepsilon^{\prime}}{k}
$$

for some integer $k$ and $\varepsilon^{\prime}= \pm 1$. Since $p>q$, we must have $k \geq 4$. Since $r=p-q \geq 2$ and $q \geq 2$, we get $p \geq q+2 \geq 4$. If $(3-k)^{2}-k \leq 0$ then we get $k=4$ or 5 since $k \geq 4$. Otherwise, we have $k \geq 6$ and

$$
4 \leq p=\frac{-3 k \varepsilon+3 \varepsilon^{\prime}}{(3-k)^{2}-k} \leq \frac{3 k+3}{(3-k)^{2}-k},
$$

and solving these inequalities, we see that $k$ must be equal to 6 . Hence we can conclude that $k$ may have three values: 4,5 or 6 .

If $k=4$, then $p=4 \varepsilon-\varepsilon^{\prime}$ and $q=3 \varepsilon-\varepsilon^{\prime}$, so $r=p-q=\varepsilon= \pm 1$, a contradiction. If $k=6$, then $p=-6 \varepsilon+\varepsilon^{\prime}$ and $q=-3 \varepsilon+\varepsilon^{\prime} / 3$, so $q$ cannot be an integer, a contradiction. Suppose $k=5$. Then $p=15 \varepsilon-3 \varepsilon^{\prime}$ and $q=9 \varepsilon-2 \varepsilon^{\prime}$, so $\varepsilon=1$ and $(p, q)=(12,7)$ or $(18,11)$ depending on whether $\varepsilon^{\prime}=1$ or -1 . Thus $K^{\prime}=T(12,7,5,-3)$ or $T(18,11,7,-3)$. This contradicts statements (2) and (4) of Lemma 3.1.

\section{The case $s=-2$}

Throughout this section, we assume $s=-2$. We begin with an elementary numbertheoretic observation.

Lemma 4.1 Any positive integral solution to the equation $x y-(x-y)^{2}= \pm 1$ is given by $x=f_{n+1}$ and $y=f_{n-1}$ up to interchanging $x$ and $y$, where $f_{k}$ is the $k^{\text {th }}$ entry of the Fibonacci sequence defined by $f_{k+1}=f_{k}+f_{k-1}\left(f_{1}=f_{2}=1\right)$.

Proof Using Cassini identity $f_{n+1} f_{n-1}-f_{n}^{2}=(-1)^{n}$, one easily verifies that $(x, y)=\left(f_{n+1}, f_{n-1}\right)\left(\right.$ or $\left.\left(f_{n-1}, f_{n+1}\right)\right)$ is a solution. 
Conversely, suppose that $\left(x_{1}, y_{1}\right)$ is a positive integral solution. Clearly, $x_{1}$ and $y_{1}$ are relatively prime. Without loss of generality, we may assume that $x_{1}>y_{1}$. Recursively define

$$
x_{k}=x_{k-1}-y_{k-1} \quad \text { and } \quad y_{k}=x_{k-1}-2 y_{k-1}
$$

for each $k \geq 2$. Straightforward induction can be used to verify that $\left(x_{k}, y_{k}\right)$ is a solution to the equation $x y-(x-y)^{2}= \pm 1$. Let $m$ be the smallest integer for which $y_{m+1} \leq 0$. By the choice of $m, x_{m}-2 y_{m}=y_{m+1} \leq 0$ and $x_{m}-y_{m}=$ $\left(x_{m-1}-y_{m-1}\right)-\left(x_{m-1}-2 y_{m-1}\right)=y_{m-1}>0$. Hence we obtain

$$
1 \leq y_{m}<x_{m} \leq 2 y_{m},
$$

so $x_{m} y_{m} \geq 2$. Since $\left(x_{m}, y_{m}\right)$ is a solution of the equation $x y-(x-y)^{2}= \pm 1$, $\left(x_{m}, y_{m}\right)$ satisfies $x_{m}^{2}+y_{m}^{2}=3 x_{m} y_{m} \pm 1$. Dividing both sides of the equation $x_{m}^{2}+y_{m}^{2}=3 x_{m} y_{m} \pm 1$ by $x_{m} y_{m}$ and noting that $t+1 / t$ is an increasing function for $1 \leq t \leq 2$, one sees that

$$
\frac{5}{2}=2+\frac{1}{2} \geq \frac{x_{m}}{y_{m}}+\frac{y_{m}}{x_{m}}=3 \pm \frac{1}{x_{m} y_{m}} \geq 3-\frac{1}{x_{m} y_{m}} \geq \frac{5}{2} .
$$

Thus $x_{m}=2=f_{3}$ and $y_{m}=1=f_{1}$.

The defining relation in Equation (4-1) can be converted into

$$
x_{k-1}=2 x_{k}-y_{k} \quad \text { and } \quad y_{k-1}=x_{k}-y_{k} .
$$

Using this relation, one can inductively deduce that $x_{m-i}=f_{i+3}$ and $y_{m-i}=f_{i+1}$ for $0 \leq i \leq m-1$. Hence $x_{1}=f_{m+2}$ and $y_{1}=f_{m}$.

Recall that we have $r=p-1$ or $p-q$ by Lemmas 2.1, 2.2 and 2.5.

Lemma 4.2 If $K^{\prime}=T(p, \varepsilon q)$, then $K^{\prime}=T(5,2,3,-2)=T(5,-2)$.

Proof Assume that $K^{\prime}=T(p, \varepsilon q)$. Then

$$
\left|p q-2 r^{2}-\varepsilon p q\right|=2 .
$$

First, suppose $r=p-1$. Then Equation (4-2) gives $(1-\varepsilon) p q \pm 2=2(p-1)^{2}$. Since $r=p-1>p-2 \geq q$,

$$
\begin{aligned}
2 p q+2 \geq(1-\varepsilon) p q \pm 2 & =2(p-1)^{2} \\
& =2 p^{2}-4 p+2 \geq 2 p(q+2)-4 p+2=2 p q+2 .
\end{aligned}
$$

Thus $\varepsilon=-1$ and $p=q+2(=r+1)$. It follows that $T(p, q, r, s)=T(r+1, r-1, r,-2)$ and $r$ is even. By an isotopy one can deform $T(r+1, r-1, r,-2)$ to the mirror image 
of the knot shown in Figure 10 (a). See Figure 10 (b), which illustrates the case that $r=4$. Thus the genus of $T(r+1, r-1, r,-2)$ is equal to

$$
\frac{1-r+\left((r-1)^{2}+2(r-2)\right)}{2}=\frac{(r+1)(r-2)}{2} .
$$

However, the genus of $T(p, \varepsilon q)$ is equal to $\frac{1}{2}(p-1)(q-1)=\frac{1}{2} r(r-2)$, a contradiction. Hence $T(p, q, r, s) \neq T(p, \varepsilon q)$.

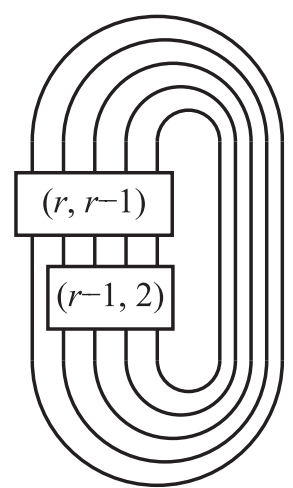

(a)

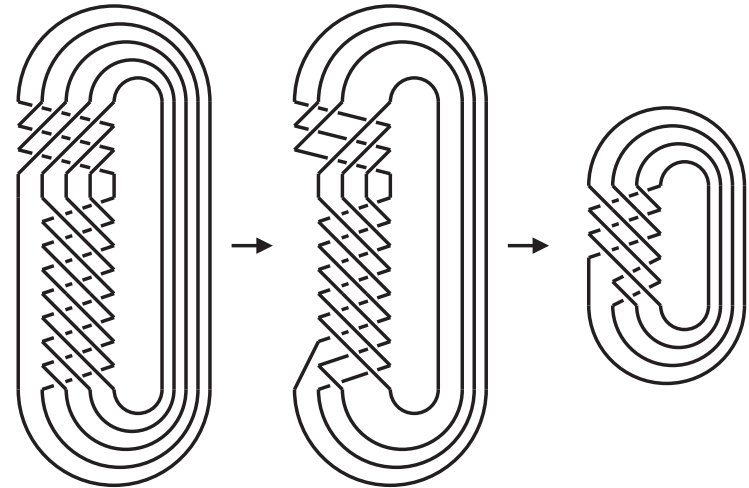

(b)

Figure 10

Next, suppose $r=p-q$. If $\varepsilon=1$, then Equation (4-2) implies $r=1$, a contradiction. Thus $\varepsilon=-1$ and Equation (4-2) gives

$$
p q-(p-q)^{2}= \pm 1
$$

By Lemma 4.1 we have $p=f_{n+1}$ and $q=f_{n-1}$ for some $n \geq 4$, where $f_{k}$ is the $k^{\text {th }}$ Fibonacci number. By Lemma 2.6 the genus $g$ of $T(p, q, r, s)$ is given by the formula

$$
\begin{aligned}
2 g & =1-(p-q)+((p-q-1)(2 p-4 q)+(p-2 q) q) \\
& =1-f_{n}+\left(f_{n}-1\right) \times 2 f_{n-2}+f_{n-2} f_{n-1} .
\end{aligned}
$$

On the other hand, the genus $g^{\prime}$ of the torus knot $T\left(f_{n+1},-f_{n-1}\right)$ is given by $2 g^{\prime}=$ $\left(f_{n+1}-1\right)\left(f_{n-1}-1\right)$. Catalan's identity states that for all positive integers $m$ and $k$ such that $m>k$ the following holds:

$$
f_{m}^{2}-f_{m+k} f_{m-k}=(-1)^{m-k} f_{k}^{2} .
$$


Using Catalan's identity, we get

$$
\begin{aligned}
2 g^{\prime}-2 g & =f_{n+1} f_{n-1}-f_{n+1}-f_{n-1}+f_{n}-2 f_{n-2}\left(f_{n}-1\right)-f_{n-2} f_{n-1} \\
& =f_{n+1} f_{n-1}-2 f_{n-1}+2 f_{n-2}-2 f_{n-2} f_{n}-f_{n-2} f_{n-1} \\
& =f_{n+1} f_{n-1}-2 f_{n-3}-2 f_{n-2} f_{n}-f_{n-2} f_{n-1} \\
& =f_{n+1} f_{n-1}-2 f_{n-3}-f_{n-2} f_{n}-f_{n-2} f_{n+1} \\
& =f_{n+1} f_{n-1}-2 f_{n-3}-f_{n-2} f_{n+2} \\
& =\left(f_{n+1} f_{n-1}-f_{n}^{2}\right)-2 f_{n-3}+\left(f_{n}^{2}-f_{n-2} f_{n+2}\right) \\
& =(-1)^{n} f_{1}^{2}-2 f_{n-3}+(-1)^{n-2} f_{2}^{2} \\
& =2(-1)^{n}-2 f_{n-3},
\end{aligned}
$$

which is equal to zero if and only if $n=4$. Thus $K^{\prime}=T(5,2,3,-2)$ and it is easy to see that $T(5,2,3,-2)=T(5,-2)$.
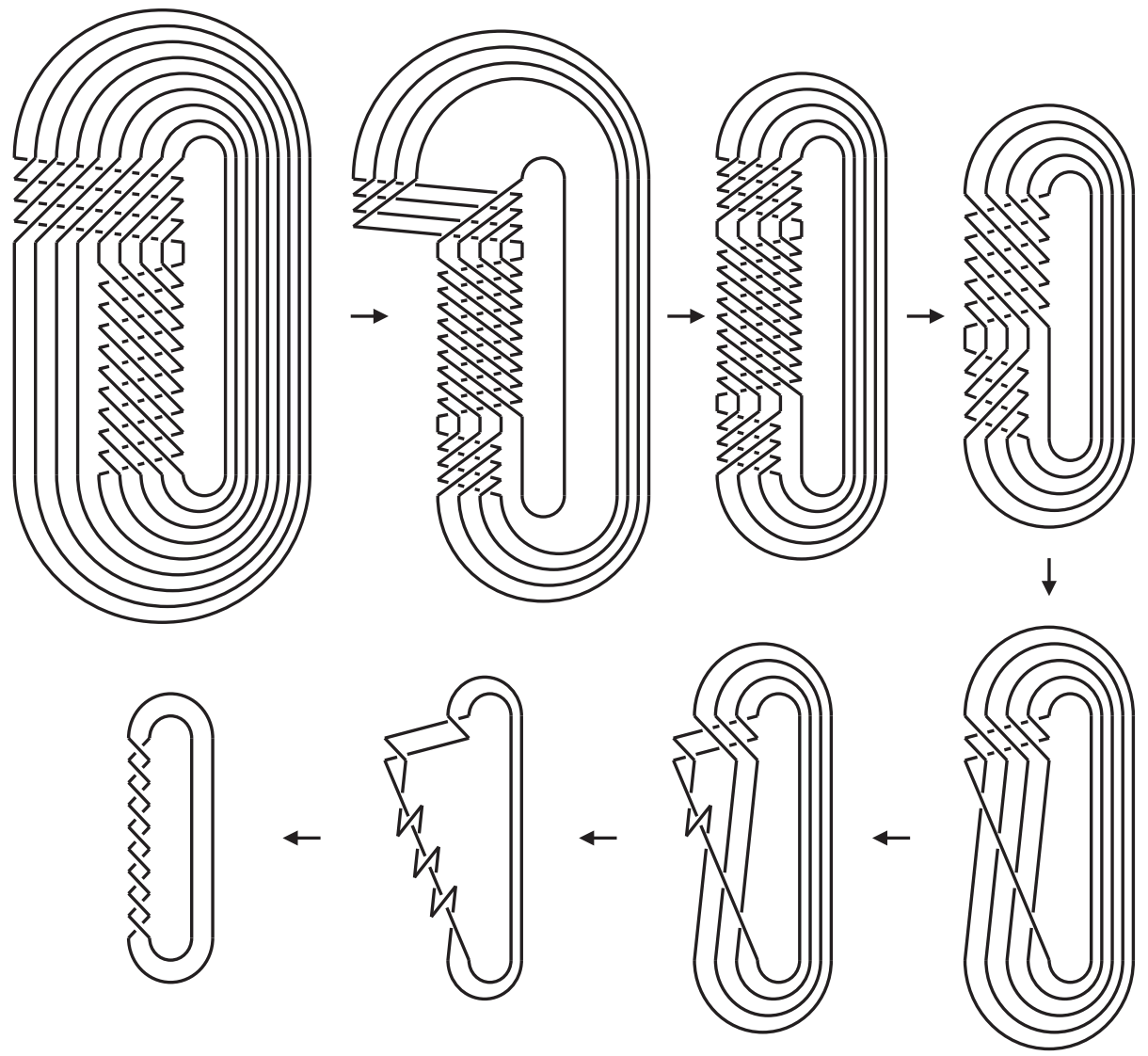

Figure 11 
Lemma 4.3 If $K^{\prime}=T(p, 2 \varepsilon)$, then $K^{\prime}=T(2 q \pm 1, q, q \pm 1,-2)=T(2 q \pm 1, \mp 2)$.

Proof Assume that $K^{\prime}=T(p, 2 \varepsilon)$. Then

$$
\left|p q-2 r^{2}-2 \varepsilon p\right|=q .
$$

First, suppose $r=p-1$. Then Equation (4-3) gives $p(2 p-4+2 \varepsilon-q)= \pm q-2$. Hence $p$ divides $q-2$ or $q+2$. In the former case, $q=2$ and $2 p-4+2 \varepsilon-q=0$, implying that $p=2$ or 4 , and this gives a contradiction because $p$ and $q$ are relatively prime. In the latter, $p=q+2$ and $2 p-4+2 \varepsilon-q=-1$, implying that $q=-1-2 \varepsilon \leq 1$, and this is also impossible because $q \geq 2$.

Next, suppose $r=p-q$. Then Equation (4-3) gives $p(2 p-5 q+2 \varepsilon)=-q(2 q \pm 1)$. Since $p$ and $q$ are relatively prime, $p$ divides $2 q \pm 1$. Since $p>q, p=2 q \pm 1$ (and $r=q \pm 1)$.

If $(p, q, r)=(2 q+1, q, q+1)$, then an isotopy deforms $T(p, q, r, s)=T(2 q+$ $1, q, q+1,-2)$ to the mirror image of $T(q+1, q+2, q,-1)$. See the upper pictures in Figure 11, which illustrates the case that $q=4$. A further isotopy deforms the mirror image to $T(2 q+1,-2)$. See the lower pictures.

If $(p, q, r)=(2 q-1, q, q-1)$, then $r<p$ and $r<q$, so we have $T(p, q, r, s)=$ $T(q, p, r, s)=T(q, 2 q-1, q-1,-2)$ by [3, Lemma 3.1.1]. By an isotopy, one can deform $T(q, 2 q-1, q-1,-2)$ to $T(2 q-1,2)$. See Figure 12, which illustrates the case that $q=5$.
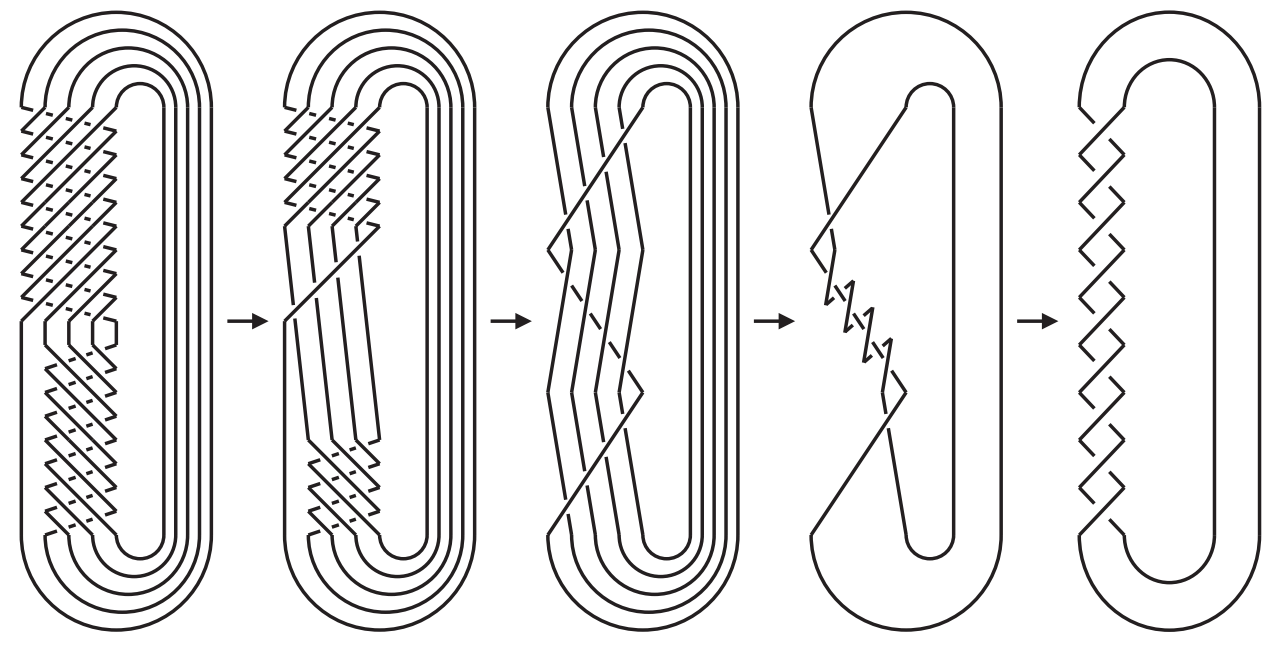

Figure 12 


\section{Lemma 4.4}

$$
K^{\prime} \neq T(q, 2 \varepsilon)
$$

Proof Assume for the sake of contradiction that $K^{\prime}=T(q, 2 \varepsilon)$. Then

$$
\left|p q-2 r^{2}-2 \varepsilon q\right|=p .
$$

First, suppose $r=p-1$. Then Equation (4-4) gives $-2(\varepsilon q+1)=p(2 p-4-q \pm 1)>$ $2 p-4-q-1 \geq 2(q+2)-4-q-1=q-1>0$ and hence $\varepsilon=-1$. Also, $p$ divides $2(q-1)$. Since $p>2$ and $p>q-1$, we must have $p=2(q-1)$ and $2 p-4-q \pm 1=1$, which yields $3 q=9 \pm 1$. This cannot happen.

Next, suppose $r=p-q$. Then Equation (4-4) gives $p(2 p-5 q \pm 1)=-2 q(q+\varepsilon)$. Since $p$ and $q$ are relatively prime, $p$ divides $2(q+\varepsilon)$ and hence $p=q+\varepsilon$ or $2 q+2 \varepsilon$. If $p=q+\varepsilon$, then $\varepsilon=1$ and $r=p-q=1$, a contradiction. If $p=2 q+2 \varepsilon$, then $2 p-5 q \pm 1=-q$ and hence $0=2 p-5 q \pm 1+q=4 \varepsilon \pm 1$, which is impossible.

Acknowledgements The author was supported by the National Research Foundation of Korea Grant funded by the Korean Government (NRF-2013R1A1A2A10064864).

\section{References}

[1] M Boileau, M Rost, H Zieschang, On Heegaard decompositions of torus knot exteriors and related Seifert fibre spaces, Math. Ann. 279 (1988) 553-581 MR922434

[2] M Cohen, W Metzler, A Zimmermann, What does a basis of $F(a, b)$ look like?, Math. Ann. 257 (1981) 435-445 MR639577

[3] J C Dean, Hyperbolic knots with small Seifert-fibered Dehn surgeries, $\mathrm{PhD}$ thesis, University of Texas at Austin (1996) MR2694392 Available at http:// search.proquest. com/docview/304274520

[4] J C Dean, Small Seifert-fibered Dehn surgery on hyperbolic knots, Algebr. Geom. Topol. 3 (2003) 435-472 MR1997325

[5] CM Gordon, Boundary slopes of punctured tori in 3-manifolds, Trans. Amer. Math. Soc. 350 (1998) 1713-1790 MR1390037

[6] C M Gordon, Y-Q Wu, Annular Dehn fillings, Comment. Math. Helv. 75 (2000) 430-456 MR1793797

[7] W Jaco, Adding a 2-handle to a 3-manifold: An application to property $R$, Proc. Amer. Math. Soc. 92 (1984) 288-292 MR754723

[8] S Lee, Twisted torus knots $T(p, q ; k q, s)$ are cable knots, J. Knot Theory Ramifications 21 (2012) MR2887898

[9] S Lee, Twisted torus knots with essential tori in their complements, J. Knot Theory Ramifications 22 (2013) MR3092503 
[10] S Lee, Twisted torus knots that are unknotted, Int. Math. Res. Not. 2014 (2014) 49584996 MR3264672

[11] Y Moriah, Heegaard splittings of Seifert fibered spaces, Invent. Math. 91 (1988) 465-481 MR928492

[12] Y Moriah, E Sedgwick, Heegaard splittings of twisted torus knots, Topology Appl. 156 (2009) 885-896 MR2498921

[13] K Morimoto, M Sakuma, On unknotting tunnels for knots, Math. Ann. 289 (1991) 143-167 MR1087243

[14] K Morimoto, M Sakuma, Y Yokota, Examples of tunnel number one knots which have the property " $1+1=3$ ", Math. Proc. Cambridge Philos. Soc. 119 (1996) 113-118 MR1356163

[15] K Morimoto, Y Yamada, A note on essential tori in the exteriors of torus knots with twists, Kobe J. Math. 26 (2009) 29-34 MR2583175

[16] D Rolfsen, Knots and links, Mathematics Lecture Series 7, Publish or Perish, Berkeley, CA (1976) MR0515288

[17] J R Stallings, Constructions of fibred knots and links, from: "Algebraic and geometric topology, Part 2", (R J Milgram, editor), Proc. Sympos. Pure Math. 32, Amer. Math. Soc. (1978) 55-60 MR520522

Department of Mathematics, Chung-Ang University 84 Heukseok-ro, Dongjak-gu, Seoul 156-756, South Korea sylee@cau.ac.kr

Received: 30 June $2014 \quad$ Revised: 4 August 2014 